\title{
D'Alembertian Solutions of Inhomogeneous Linear Equations (differential, difference, and some other) *
}

\author{
Sergei A. Abramov \\ Computer Center of \\ the Russian Academy of Science, \\ Vavilova 40, Moscow 117967, Russia. \\ abramov@ccas.ru
}

\author{
Eugene V. Zima \\ Dept. of Computat. Math. \& Cybernetics, \\ Moscow State University, \\ Moscow 119899, Russia. \\ zima@cs.msu.su
}

\section{Abstract}

Let an Ore polynomial ring $k[X ; \sigma, \delta]$ and a nonzero pseudolinear map $\theta: K \rightarrow K$, where $K$ is a $\sigma, \delta$-compatible extension of the field $k$, be given. Then we have the ring $k[\theta]$ of operators $K \rightarrow K$. It is assumed that if a first-order equation $F y=0, F \in k[\theta]$, has a nonzero solution in a $\sigma, \delta$-compatible extension of the field $k$, then the equation has a nonzero solution in $K$. These solutions form the set $\mathcal{H}_{k} \subset K$ of hyperexponential elements. An equation $P y=0, P \in k[\theta]$, is called completely factorable if $P$ can be decomposed in the product of first-order operators over $k$. Solutions of all completely factorable equations form the linear space $\mathcal{A}_{k} \subset K$ of d'Alembertian elements. The order of minimal operator over $k$ which annihilates $a \in \mathcal{A}_{k}$ is called the height of $a$. It is easy to see that $\mathcal{H}_{k} \subset \mathcal{A}_{k}$ and the height of any $a \in \mathcal{H}_{k}$ is equal to 1 .

It is known $([12,4])$ that if $L \in k[\theta]$ and $f \in \mathcal{H}_{k}$ then all the hyperexponential solutions of the equation

$$
L y=f
$$

have the form $u f, u \in k$. Substitution $y=u f$, where $u$ is a new unknown, gives us the equation

$$
M u=g, \quad M \in k[\theta], \quad g \in k . \quad(* *)
$$

If all the solutions of $\left(^{* *}\right)$ in $k$ are found, then all the hyperexponential solutions of $\left({ }^{*}\right)$ are found as well. The problem of solving in $k$ an equation whose coefficients and the right-hand side belong to $k$, is called the $k$-problem. Fast algorithms to solve $k$-problems are known for some concrete Ore polynomial rings (e.g., such algorithms for linear ordinary differential and $(q-)$ difference equations with rational functions coefficients and right-hand sides have been given in $[1,2,4])$. They allow us to find hyperexponential solutions of the equation $(*)$ quickly.

We consider in this paper the search for d'Alembertian solutions of an equation of the form (*) with $f \in \mathcal{A}_{k}$ (the height of $f$ is $r \geq 1$ ). We show that in general case the

\footnotetext{
*Work reported herein was supported in part by the RFBR (Russia) under Grant 95-01-01138a.

Permission to make digital/hard copies of all or part of this material for personal or classroom use is granted without fee provided that the copies are not made or distributed for profit or commercial advantage, the copyright notice, the title of the publication and its date appear, and notice is given that copyright is by permission of the $A C M$, Inc. To copy otherwise, to republish, to post on servers or to redistribute to lists, requires specific permission and/or fee. ISSAC'96, Zurich, Switzerland; (C)1996 ACM 0-89791-796-0/96/07. . \$3.50
}

search can be reduced to $k$-problems and to the search for hyperexponential solutions of homogeneous equations of the order $\leq$ ord $L$ over $k$. If the equation $L y=0$ has no hyperexponential solution then the search for d'Alembertian solutions of $\left(^{*}\right)$ can be reduced only to $k$-problems. If $\left(^{*}\right)$ has a solution of the height $r$, then solving only $k$-problems one can find a solution of the height $\geq r$. We describe some algorithms and an implementation in Maple 5.3 of one of them. The implementation is oriented towards an arbitrary Ore polynomial ring and can be adapted, for example, to the differential and $(q-)$ difference cases.

\section{Basic notions and formulation of problem}

Linear inhomogeneous equations of the form

$$
L y=f
$$

which we will consider can be, for example, ordinary differential, difference or $q$-difference equations. The general approach to such equations is possible in the frame of pseudolinear algebra ([9]) which has been formed on the base of Ore polynomial rings theory ([11]). Considering equation (1) we assume that $L$ is an operator of the form $p(\theta)$, where $p(X)$ belongs to Ore polynomial ring $k[X ; \sigma, \delta]$, and $\theta$ is a pseudo-linear map $K \rightarrow K$ of a $\sigma, \delta$-compatible extension ring $K$ of the field $k$. Here $k$ is a field of characteristic $0, X$ is an indeterminate over $k, \sigma$ is an automorphism of $k$ and $\delta: k \rightarrow k$ is a map satisfying

$$
\delta(a+b)=\delta a+\delta b, \quad \delta(a b)=\sigma(a) \delta b+\delta a b ;
$$

in turn, $\theta$ satisfies

$$
\theta(a+b)=\theta a+\theta b, \quad \theta(a b)=\sigma(a) \theta b+\delta a b .
$$

We will denote by $\operatorname{Const}(K)$ the constant subring of $K$ (i.e. the set of all $a \in K$ such that $\sigma(a)=a, \delta a=0$ ) and assume that $K$ is such that its constant subring is a field.

We can consider the ring $k[\theta]$ of operators $K \rightarrow K$ of the form $p(\theta), p(X) \in k[X ; \sigma, \delta]$. These operators are linear over Const $(K)$.

It is assumed that if a first-order equation $F y=0, F \in$ $k[\theta]$, has a nonzero solution in a $\sigma, \delta$-compatible extension of the field $k$, then the equation has a nonzero solution in $K$. These solutions form the set $\mathcal{H}_{k} \subset K$ of hyperexponential elements. An equation $P y=0$ and the operator $P$ are called completely factorable if $P$ can be decomposed in the product of first-order operators over $k$. Solutions of all completely factorable equations form the linear space $\mathcal{A}_{k} \subset K$ of d'Alembertian elements. It is easy to see that $\mathcal{H}_{k} \subset \mathcal{A}_{k}$. 
Let $L \in k[\theta]$ and $S$ be the space of solutions of $L y=0$ belonging to $K$. We assume that $\operatorname{dim} S \leq \operatorname{ord} L$ in such situation.

The following notions generalize those that have been used in [3] for an investigation of d'Alembertian solutions of differential and difference equations:

- the operator $\nabla$, which is an analog of $\frac{d}{d x}$ and $\Delta$ :

$$
\nabla= \begin{cases}\delta, & \text { if } \sigma=1 \\ \sigma-1, & \text { if } \sigma \neq 1\end{cases}
$$

It is easy to show that $\nabla f=0 \Leftrightarrow f \in \operatorname{Const}(K)$

- the set $I(f), f \in K$, which is an analog of the indefinite integral and sum:

$$
I(f)=\{d \mid \nabla d=f\} .
$$

If $f \in K, d \in I(f), c_{0} \in C o n s t(K)$, then $d+c_{0} \in I(f)$ and, vice versa, for any $d_{1}, d_{2} \in I(f)$ we have $d_{1}-d_{2} \in$ Const $(K)$. We assume that $I(f)$ is not empty for any $f \in K$. If a d'Alembertian space $A$ is given, then we can construct the completely factorable operator over $k$ such that $A$ is its solution space. If $\mathcal{U}$ is a set of elements of $K$, then $I(\mathcal{U})$ denotes the set of all $d$ such that $\nabla d \in \mathcal{U}$. We write for brevity $I\left(f_{1}, \ldots, f_{m}\right)$ instead of $f_{1} I\left(f_{2} I\left(f_{3} \ldots f_{m-1} I\left(f_{m}\right) \ldots\right)\right)$;

- d'Alembert substitution

$$
y=\varphi I(u)
$$

connected with a solution $\varphi$ of a linear equation $L y=$ 0 . This substitution reduces the order of the equation. The search for d'Alembertian solutions can be reduced to the search for hyperexponential solutions and to d'Alembert substitutions;

- d'Alembertian space

$$
I\left(\varphi_{1}, \ldots, \varphi_{r}, 0\right),
$$

where $\varphi_{1}, \ldots, \varphi_{r} \in \mathcal{H}_{k}$. Any d'Alembertian space is the solution space of a completely factorable operator. It is easy to see that

$$
I\left(\varphi_{1}, 0\right) \subset I\left(\varphi_{1}, \varphi_{2}, 0\right) \subset \ldots \subset I\left(\varphi_{1}, \ldots, \varphi_{r}, 0\right) .
$$

Let $k=\mathbf{C}(x), \sigma=1$ (the identity authomorphism), $\delta=\theta=d / d x$. Then $\sin x \in \mathcal{A}_{k}$, because

$$
\sin x=\frac{e^{\imath x}-e^{-\imath x}}{2 i} \in e^{\imath x} \int e^{-2 i x} \int 0=I\left(e^{\imath x}, e^{-2 i x}, 0\right) .
$$

We can define concretely the integrals for $\sin x$ :

$$
e^{i x}\left(\int_{0}^{x} e^{-2 i t}\left(\int_{0}^{t} 0+1\right)+0\right)=e^{\imath x} \int_{0}^{x} e^{-2 \imath t} ;
$$

$\sin x$ satisfies the completely factorable equation $y^{\prime \prime}+y=0$.

Let $k=\mathbf{C}(x), \sigma=\theta=E$ (the shift operator), $\delta=0$. Observe that $E$ is not an authomorphism of a ring of sequences, since it annihilates nonzero sequence of the form $(c, 0,0, \ldots), c \neq 0$. But we can identify any two sequences which agree from some point on. To simplify notation, we will identify a class $\{u\}$ of equivalent sequences with its representative sequence $u$.
For Fibonacci numbers $u_{m}$ we have $u_{m} \in \mathcal{A}_{k}$ because

$$
\begin{aligned}
& u_{m}=\frac{1}{\sqrt{5}}\left(\left(\frac{1+\sqrt{5}}{2}\right)^{m}-\left(\frac{1-\sqrt{5}}{2}\right)^{m}\right) \epsilon \\
& \epsilon\left(\frac{1+\sqrt{5}}{2}\right)^{m} \sum\left(\frac{1-\sqrt{5}}{1+\sqrt{5}}\right)^{m} \sum 0= \\
& =I\left(\left(\frac{1+\sqrt{5}}{2}\right)^{m},\left(\frac{1-\sqrt{5}}{1+\sqrt{5}}\right)^{m}, 0\right) .
\end{aligned}
$$

We can define concretely the sums for $u_{m}$ :

$$
\begin{aligned}
& \left(\frac{1+\sqrt{5}}{2}\right)^{m}\left(\sum_{l=0}^{m-1}\left(\frac{1-\sqrt{5}}{1+\sqrt{5}}\right)^{l}\left(\sum_{\nu=0}^{l-1} 0+\frac{2}{1+\sqrt{5}}\right)+0\right)= \\
& =\left(\frac{1+\sqrt{5}}{2}\right)^{m} \sum_{l=0}^{m-1} \frac{2}{1+\sqrt{5}}\left(\frac{1-\sqrt{5}}{1+\sqrt{5}}\right)^{l}
\end{aligned}
$$

(if the upper bound of a sum is less than the lower one then the sum is equal to zero). The sequence of Fibonacci numbers satisfies the completely factorable equation $E^{2} y-$ $E y-y=0$.

It can be shown that the sum and the product of any two d'Alembertian elements are d'Alembertian themselves. So, $\mathcal{A}_{k}$ is a ring, all hyperexponential elements are invertible in the ring. The consideration of the difference case shows that $\mathcal{A}_{k}$ can have zero divisors. Let $a_{m}=1+(-1)^{m}, b_{m}=$ $1-(-1)^{m}$. These sequences satisfy equation $E^{2} y-y=0$, therefore $a_{m}, b_{m} \in \mathcal{A}_{k}$. But $a_{m} b_{m}=0$.

For any two nonzero operators $L, M \in k[\theta]$ one can compute their greatest common right divisor $\operatorname{gcrd}(L, M) \in k[\theta]$. If at least one of $L$ and $M$ is completely factorable then $\operatorname{gcrd}(L, M)$ is completely factorable too.

It is known ([3]) that if $L \in k[\theta], a \in \mathcal{A}_{k}, \varphi \in \mathcal{H}_{k}$ then $L a \in \mathcal{A}_{k}$ and there exists $v \in k$ such that $L \varphi=v \varphi \in$ $\mathcal{H}_{k}$; additionally the following properties of d'Alembertian elements take place:

dA1. Let the equation $L y=0, L \in k[\theta]$, have a solution in $\mathcal{A}_{k}$. Then this equation has a solution in $\mathcal{H}_{k}$.

dA2. Let $A=I\left(\varphi_{1}, \ldots, \varphi_{r}, 0\right)$, where $\varphi_{1}, \ldots, \varphi_{r} \in \mathcal{H}_{k}$. Let $\xi_{1} \in A \cap \mathcal{H}_{k}$. Then there exist $\xi_{2}, \ldots, \xi_{r} \in \mathcal{H}_{k}$ such that $A=I\left(\xi_{1}, \ldots, \xi_{r}, 0\right)$.

Considering equation (1) we will suppose that $f \in \mathcal{A}_{k}$ and that the minimal d'Alembertian space in which $f$ can be expressed (the envelope of $f$ ) is given. If we know the envelope of $f$ then it is easy to construct minimal annihilating operator $P \in k[\theta]$ for $f$ (and vice versa, but such construction requires the search for hyperexponential solutions of homogeneous equations). Further we will denote the envelope of $f$ by $\mathcal{E}(f)$. It is easy to show that the envelope of a nonzero d'Alembertian element $f$ is unique, and that the minimal annihilating operator is unique up to a nonzero factor from $k$. The dimension of $\mathcal{E}(f)$ is called the height of $f$ (it is equal to the order of minimal annihilating operator) and will be denoted by $h(f)$. The height of the zero element is equal to 0.

When working with a d'Alembertian element $f$, we consider its envelope and minimal annihilating operator. This is analogous to working with algebraic numbers and functions, when we consider the corresponding extensions of the ground field and their minimal polynomials.

The problem of finding all d'Alembertian solutions of equation (1) has been considered in [7] for differential, difference and $q$-difference equations. This problem has been solved with the help of the standard method of transition from equation (1) to the homogeneous equation

$$
P L y=0
$$

( $P$ is the minimal annihilating operator for $f$ ). Any solution of equation (1) satisfies equation (3) and for any solution $y$ of 
equation (3) we have $L y=\tilde{f}$, where $P \tilde{f}=0$. Let ord $L=n$ and $h(f)=r$. It is sufficient to find the space $A$ of dimension $s \leq n+r$ of all d'Alembertian solutions of equation (3) and choose those solutions from $A$ which satisfy (1). This choice is described in [7] differently for each of the three abovementioned types of equations. But, in fact, it can be reduced to equating the values of $\nabla^{l} y$ and $\nabla^{l} f$ for $l=0, \ldots, s-1$ at a point $p$ which is nonsingular for the equations considered (in the differential case this is equivalent to consideration of the first terms of Taylor expansion in the neighborhood of a nonsingular point).

The standard method just mentioned requires the search for hyperexponential solutions of homogeneous equations whose order greater than the order of the initial equation. When $k$ is the field of rational functions, algorithms to find hyperexponential solutions of differential ([8]), difference ([12]) and $q$-difference ([6]) equations are known. All these algorithms are quite laborious: they require, for example, the complete factorization of polynomials, separate considerations of several hypothesis about the form of solution; it is necessary to work with algebraic numbers even if $k=\mathbf{Q}(x)$ or $k=\mathbf{Q}(q, x)$.

The goal of this work is to minimize the search for hyperexponential solutions of homogeneous equations in the process of finding d'Alembertian solution of (1). We will propose some algorithms which have some advantages over the standard method. However using the standard method let us easily prove one important feature of d'Alembertian solutions.

Proposition 1 Let $f$ on the right of (1) be of height $r$. Let (1) have a solution $a \in \mathcal{A}_{k}$ such that $h(a)>r$. Then the homogeneous equation $L y=0$ corresponding to (1) has a solution in $\mathcal{H}_{k}$.

Proof: Consider the space $A$ constructed by the standard method. The fact that $h(a)$ is greater then $r$ means that $\operatorname{dim} A>r$. Hence, it is possible to find $a_{1}, a_{2} \in A$ such that $a_{1} \neq a_{2}$ and $L a_{1}=L a_{2}$. The latter equation means that $L\left(a_{1}-a_{2}\right)=0$. With the help of $\mathbf{d A 1}$ we get the desired conclusion.

A possible approach to the construction of d'Alembertian solutions of equation (1) is based on the following fact. Let $f \in \mathcal{H}_{k}$. If (1) has a solution in $\mathcal{H}_{k}$, then this solution must be of the form $u f, u \in k$. After the substitution $y=u f$, where $u$ is a new unknown, and simplifications we get

$$
M u=g, M \in k[\theta], g \in k .
$$

Thus the question about existence in $\mathcal{H}_{k}$ of solutions of the initial equation is reduced to the question about existence in $k$ of solutions of equation (4). This fact was initially stated in [12] for difference equations and was than generalized to the case of an arbitrary Ore ring in [4]. We will call the $k$-problem the problem of searching in $k$ for solutions of an equation of the form (4). Hence, the search in $\mathcal{H}_{k}$ for solutions of equation (1) with $f \in \mathcal{H}_{k}$ is reduced to a $k$-problem. There exist fast algorithms to solve $k$-problems for differential, difference and $q$-difference equations when $k$ is the field of rational functions $([1,4,5])$. To generalize this approach (which can be called the method of reduction to $k$-problems) to the case of d'Alembertian right-hand side, we will discuss in detail the following task:

T. Let (1) have a d'Alembertian solution and $\mathcal{E}(f)$ be of the form

$$
I\left(\psi_{1}, \ldots, \psi_{r}, 0\right)
$$

$\psi_{1}, \ldots, \psi_{r} \in \mathcal{H}_{k}$. Decide whether the equation

$$
L Y=\mathcal{E}(f)
$$

has a solution in the class of d'Alembertian spaces, or not, i.e. whether there is a d'Alembertian space $A$ of the form

$$
\begin{gathered}
I\left(\varphi_{1}, \ldots, \varphi_{s}, 0\right), \\
\varphi_{1}, \ldots, \varphi_{s} \in \mathcal{H}_{k}, \text { such that } \\
L A=\mathcal{E}(f),
\end{gathered}
$$

or not. If $A$ exists, construct $\varphi_{1}, \ldots, \varphi_{s}$.

Obviously, if (8) takes place, then $s \geq r$. If $s>r$ then the homogeneous equation of order $n$

$$
L y=0
$$

has a hyperexponential solution. We will show that the search for some spaces $A$ (and for the maximal $A$ ) satisfying (8) can be reduced to $k$-problems and to the search for hyperexponential solutions of homogeneous equations of order $\leq n$. The corresponding algorithms will be given in details. With additional assumptions about equation (1) or (8) one needs no search for hyperexponential solutions of homogeneous equations (some algorithms of this kind will be described).

Equation (1) is of the main interest to us. We will show further that if (1) has a solution $a \in \mathcal{A}_{k}$ then for some d'Alembertian space $A$ equation (8) is satisfied. Therefore solving equation (1) can be reduced to the task $\mathbf{T}$ and to the search in $A$ for an element satisfying (1).

\section{Equations in the class of d'Alembertian spaces}

First of all we clarify how to apply the operator $L \in k[\theta]$ to the space $A$ of the form ( 7 ) defined by $\varphi_{1}, \ldots, \varphi_{r}$. We will see that the result of this application is a d'Alembertian space again. Below, we will describe the process of this application recursively, reducing it to the application of another operator to the space $I\left(\varphi_{2}, \ldots, \varphi_{r}, 0\right)$. In order to describe this reduction more concisely, we define the operator $L^{[\varphi]} \in k[\theta]$ for $\varphi \in \mathcal{H}_{k}$ and $L \in k[\theta]$. Consider $T=L \circ \varphi$ (we use the sign $\circ$ here having in mind the product of operators $L$ and $\varphi$ ). It is easy to show that

$$
T=\bar{T} \nabla+L \varphi
$$

where $\bar{T}$ is some operator with coefficients from $\mathcal{H}_{k}$ and $L \varphi$ means as usually the result of applying $L$ to $\varphi$. Let

$$
\tilde{T}= \begin{cases}\frac{1}{\varphi} T, & \text { if } L \varphi=0, \\ \nabla \frac{1}{L \varphi} T, & \text { if } L \varphi \neq 0 .\end{cases}
$$

In any case $\tilde{T} \in k[\theta]$. We define $L^{[\varphi]}$ from the operator equality

$$
\tilde{T}=L^{[\varphi]} \nabla,
$$

which is solvable in $k[\theta]$ because of (10). We can see that

$$
\operatorname{ord} L^{[\varphi]}= \begin{cases}\operatorname{ord} L, & \text { if } L \varphi \neq 0 \\ \operatorname{ord} L-1, & \text { if } L \varphi=0\end{cases}
$$

A practical way to construct $L^{[\varphi]}$ is the following. Compute $\tilde{T} \in k[\theta]$ according to (11) and take the right quotient of $\tilde{T}$ by $\nabla$ (for this purpose the operator $\nabla$ has to be represented as an element of $k[\theta])$. 
Using operators of the form $L^{[\varphi]}$ we can prove by induction on $s$ that $L I\left(\varphi_{1}, \ldots, \varphi_{s}, 0\right)$ is a d'Alembertian space and describe the process of its construction. We will concentrate on the process of construction. Let

$$
L^{\left[\varphi_{1}\right]} I\left(\varphi_{2}, \ldots, \varphi_{s}, 0\right)=I\left(\xi_{1}, \ldots, \xi_{t}, 0\right),
$$

where $\xi_{1}, \ldots, \xi_{t} \in \mathcal{H}_{k}, t \geq 0$. By definition of $L^{\left[\varphi_{1}\right]}$ we have

$$
\begin{aligned}
& L I\left(\varphi_{1}, \ldots, \varphi_{s}, 0\right)=I\left(L \varphi_{1}, \xi_{1}, \ldots, \xi_{t}, 0\right), \\
& \text { if } L \varphi_{1} \neq 0, \\
& L I\left(\varphi_{1}, \ldots, \varphi_{s}, 0\right)=I\left(\varphi_{1} \xi_{1}, \xi_{2}, \ldots, \xi_{t}, 0\right), \\
& \text { if } L \varphi_{1}=0 .
\end{aligned}
$$

Proposition 2 Let $\varphi_{1}, \ldots, \varphi_{s}, \psi_{1}, \ldots, \psi_{r} \in \mathcal{H}_{k}, s \geq r$. Let $L \in k[\theta]$.

If $L \varphi_{1}=\psi_{1}$, then

$$
\begin{aligned}
& L I\left(\varphi_{1}, \ldots, \varphi_{s}, 0\right)=I\left(\psi_{1}, \ldots, \psi_{r}, 0\right) \Leftrightarrow \\
& \Leftrightarrow L^{\left[\varphi_{1}\right]} I\left(\varphi_{2}, \ldots, \varphi_{s}, 0\right)=I\left(\psi_{2}, \ldots, \psi_{r}, 0\right) .
\end{aligned}
$$

If $L \varphi_{1}=0$, then

$$
\begin{aligned}
& L I\left(\varphi_{1}, \ldots, \varphi_{s}, 0\right)=I\left(\psi_{1}, \ldots, \psi_{r}, 0\right) \Leftrightarrow \\
& \Leftrightarrow L^{\left[\varphi_{1}\right]} I\left(\varphi_{2}, \ldots, \varphi_{s}, 0\right)=I\left(\psi_{1} / \varphi_{1}, \psi_{2}, \ldots, \psi_{r}, 0\right) .
\end{aligned}
$$

Proof: In both the cases

$$
\Rightarrow \text { : by definition of } L^{\left[\varphi_{1}\right]} \text {. }
$$

$\Leftarrow$ : with the help of (12).

Observe that in the case where $L \varphi_{1}=0$ the transformation of (9) into $L^{\left[\varphi_{1}\right]} y=0$, or of (1) into $L^{\left[\varphi_{1}\right]} y=f / \varphi_{1}$, is equivalent to D'Alembert substitution (2).

Proposition 3 Let (1) have a solution a $\in \mathcal{A}_{k}$ such that $f$ and $a$ have the same herght $r$. Let $A=\mathcal{E}(a)$ and $B=\mathcal{E}(f)$. Then $L A=B$.

Proof: The space $L A$ is d'Alembertian, $\operatorname{dim} L A \leq r$. The space $L A \cap B$ is not empty, because $f \in L A \cap B$. This intersection is a d'Alembertian space (it follows from the existence of gcrd in $k[\theta])$. It is obvious that if $L A \neq B$ then $\operatorname{dim}(L A \cap B)<r$ which contradicts the fact that $B=\mathcal{E}(f)$.

Proposition 4 Suppose that equation (1) has a solution $a \in \mathcal{A}_{k}$ while equation (9) has no solution in $\mathcal{H}_{k}$. Then the space $A=\mathcal{E}(a)(\operatorname{dim} A=r)$ is the unique solution of equation (6) in the class of d'Alembertian spaces. If $a_{1} \in \mathcal{A}_{k}$ and $a_{1} \notin A$ then $L a_{1} \notin B$.

Proof: Due to Proposition 1, $h(a)=r$. The space $A$ is a solution of equation (6) due to Proposition 3. Finally, equation (6) has no solutions except for $A$ because otherwise it would be possible to find two different d'Alembertian elements $a_{1}, a_{2}$ such that $L a_{1}=L a_{2}$. The latter equation means that $a_{1}-a_{2}$ solves (9). But $a_{1}-a_{2} \in \mathcal{A}_{k}$ and equation (9) has solution in $\mathcal{H}_{k}$ due to dA1. Contradiction. $\square$

Let (9) have a solution $\varphi_{1} \in \mathcal{H}_{k}$. Transform (9) into $L^{\left[\varphi_{1}\right]} y=0$. Assume the new equation is transformed again using its solution $\varphi_{2}$ and so on, until the last d'Alembert substitution connected with $\varphi_{m}$ produces the equation $L^{\left[\varphi_{1}\right] \ldots\left[\varphi_{m}\right]} y=0\left(L_{m} y=0\right.$, for brevity) with no solution in $\mathcal{H}_{k}$. It is known ([3]) that the operator $L_{m}$ is unique up to a nonzero factor from $k$.

Inhomogeneous equation (1) (for which (9) is the corresponding homogeneous) will be transformed in this way to

$$
L_{m} y=\frac{f}{\varphi_{1} \ldots \varphi_{m}}
$$

The following proposition does not depend on the existence of solutions of (9) in $\mathcal{H}_{k}$.

Proposition 5 Let (1) have a solution $a \in \mathcal{A}_{k}$. Let $B=$ $\mathcal{E}(f)$. Then the set

$$
D=\left\{d \mid d \in \mathcal{A}_{k}, L d \in B\right\}
$$

is a d'Alembertian space.

Proof: Assume that (9) has been transformed with the help of $\varphi_{1}, \ldots, \varphi_{m}$ as described above, and let equation (1) be converted to (15) (if the initial equation $L y=0$ had no solution in $\mathcal{H}_{k}$ then $m=0$ and $\left.L_{m}=L\right)$. Let $B=I\left(\psi_{1}, \ldots, \psi_{r}\right)$ be $\mathcal{E}(f)$. The space

$$
\tilde{B}=\frac{1}{\varphi_{1} \ldots \varphi_{m}} B=I\left(\frac{\psi_{1}}{\varphi_{1} \ldots \varphi_{m}}, \psi_{2}, \ldots, \psi_{r}, 0\right)
$$

is equal to $\mathcal{E}(\tilde{f})$, where $\tilde{f}$ is the right-hand side of $(15)$, i.e., $f /\left(\varphi_{1} \ldots \varphi_{m}\right)$, because if $P$ is a minimal annihilating operator for $f$ then the operator

$$
\varphi_{1} \ldots \varphi_{m} P \circ \frac{1}{\varphi_{1} \ldots \varphi_{m}}
$$

is a minimal annihilating operator for $\tilde{f}$. Due to Proposition 4 the equation $L_{m} Y=\tilde{B}$ has the unique solution $G=I\left(\xi_{1}, \ldots, \xi_{r}, 0\right)$ in the class of d'Alembertian spaces and if $d \notin G$ then $L_{m} d \notin \tilde{B}$. Therefore

$$
D=I\left(\varphi_{1}, \ldots, \varphi_{m}, \xi_{1}, \ldots, \xi_{r}, 0\right)
$$

Corollary 1 If (1) has a solution ain $\mathcal{A}_{k}$ then (6) has a solution in the class of d'Alembertian spaces (for example, space (16)). Any space which satisfies (6) includes a solution of (1).

It is easy to see that if $L \varphi_{1}=\psi_{1}$ then $\nabla\left(a / \varphi_{1}\right)$ is a d'Alembertian solution of $L^{\left[\varphi_{1}\right]}=\nabla\left(f / \psi_{1}\right)$ and $I\left(\psi_{2}, \ldots, \psi_{r}, 0\right)=\mathcal{E}\left(\nabla\left(f / \psi_{1}\right)\right)$. If $L \varphi_{1}=0$ then $\nabla\left(a / \varphi_{1}\right)$ is a d'Alembertian solution of $L^{\left[\varphi_{1}\right]}=f / \psi_{\mathrm{l}}$, and $I\left(\psi_{1} / \varphi_{1}, \psi_{2}, \ldots, \psi_{r}, 0\right)=\mathcal{E}\left(f / \psi_{1}\right)$. In both the cases we obtain simpler equation $L^{\left[\varphi_{1}\right]} Y=B_{1}$, where $B_{1}$ is $I\left(\psi_{2}, \ldots, \psi_{r}, 0\right)$ or $I\left(\psi_{1} / \varphi_{1}, \psi_{2}, \ldots, \psi_{r}, 0\right)$ respectively, instead of equation $L Y=B$ of the form (6).

Thanks to Corollary 1 we have the following Corollary 2 Let (1) have a solution a $\in \mathcal{A}_{k}$. Let $B=\mathcal{E}(f)$ in the equation $L Y=B$. Let this equation be transformed into $L^{\left[\varphi_{1}\right] \cdot\left[\varphi_{m}\right]} Y=B_{m}$ by transformations described above. Then the last equation has a solution in the class of d'Alembertian spaces. If we know a solution of this kind then we can use formulae (13), (14) and get a solution of $L Y=B$.

Proposition 6 Let (9) have no solution in $\mathcal{H}_{k}$. Let the equation $L Y=I\left(\psi_{1}, \ldots, \psi_{r}, 0\right)$ have a solution $A$ in the class of d'Alembertian spaces. Then the equation $L y=\psi_{1}$ has a solution in $\mathcal{H}_{k}$.

Proof: There exists $a \in A$ such that $L a=\psi_{1}$. It follows from Proposition 4, that $\operatorname{dim} \mathcal{E}(a)=\operatorname{dim} \mathcal{E}\left(\psi_{1}\right)=1$. Therefore $a \in \mathcal{H}_{k}$

Corollary 3 Let (1) have a d'Alembertian solution and $\mathcal{E}(f)=I\left(\psi_{1}, \ldots, \psi_{r}, 0\right)$. Then at least one of equations $L y=0, L y=\psi_{1}$ has a solution in $\mathcal{H}_{k}$.

Corollaries 1-3 are the key results of this section. They give a base for designing algorithms to find solutions of (6) and of (1). 


\section{Algorithms}

We consider equations (1), (6) with $\mathcal{E}(f)$ given in the form (5). Our goal is the minimization of the search for solutions in $\mathcal{H}_{k}$ of homogeneous equations like (9) in the process of solving equation (6). If (9) has no solutions in $\mathcal{H}_{k}$ then one can use a very simple algorithm. In its description we will use the operations head $(B)$ and $\operatorname{tail}(B)$ : if $B$ is given in the form (5), then head $(B)=\psi_{1}, \operatorname{tail}(B)=I\left(\psi_{2}, \ldots, \psi_{r}, 0\right)$.

Observe that the representation of d'Alembertian space in form (5) is not unique, but the algorithm below treats (5) as a fixed collection of elements $\psi_{1}, \ldots, \psi_{r} \in \mathcal{H}_{k}$. Strictly speaking, the operations head and tail are operations not over spaces, but over these collections of elements.

If $\tau \in \mathcal{H}_{k}$ then $\frac{1}{\tau} B$ will denote $I\left(\frac{\psi_{1}}{\tau}, \psi_{2}, \ldots, \psi_{r}, 0\right)$.

Our algorithm is applied to

$$
L, B, \quad r
$$

where $L \in k[\theta], B=\mathcal{E}(f)$ is of the form (5), $r=\operatorname{dim} B$.

Algorithm 1

1. If ord $L=0$, i.e., $L$ is some $q \in k$, then the result will be $\frac{1}{q} B$.

2. Let $\psi=\operatorname{head}(B)$. Reduce the equation $L y=\psi$ to a $k$-problem. If the $k$-problem has no solution, the algorithm stops with the result "no solution".

3. Let $u \in k$ be a solution of $k$-problem. Let $\varphi=u \psi$. If $r=1$ then the result will be $I(\varphi, 0)$, else apply the algorithm recursively to

$$
L^{[\varphi]}, \operatorname{tail}(B), r-1 .
$$

4. If the result of the recursive call was "no solution" then the final result will be the same. Otherwise, if the result of the recursive call was a d'Alembertian space $I\left(\xi_{1}, \ldots, \xi_{t}, 0\right)$, then the final result will be $I\left(\varphi, \xi_{1}, \ldots, \xi_{t}, 0\right)$.

Consider as an example the equation of the form (6)

$$
\left(\frac{d^{2}}{d x^{2}}-x\right) Y=\frac{4 x^{3}+1}{x^{3 / 2}} \int \frac{1}{x} \int 0
$$

with $k=\mathbf{C}(x)$.

Here $L=\frac{d^{2}}{d x^{2}}-x$. The equation $L y=0$ has no solution in $\mathcal{H}_{k}$. Reduction of the equation $L y=\psi_{1}$, where $\psi_{1}=\frac{4 x^{3}+1}{x^{3 / 2}}$ to a $k$-problem gives us

$$
u^{\prime \prime}(x)+\frac{3\left(4 x^{3}-1\right)}{x\left(4 x^{3}+1\right)} u^{\prime}(x)-\frac{16 x^{6}-8 x^{3}-15}{4 x^{2}\left(4 x^{3}+1\right)} u(x)=1 .
$$

This equation has the unique solution $u=-4 x^{2} /\left(4 x^{3}+1\right)$ in $k$, which is why $\varphi_{1}=u \psi_{1}=-4 \sqrt{x}$. Further computations give us the $k$-problem

$$
-\frac{4 x^{2}}{4 x^{3}+1} u^{\prime \prime}(x)+\frac{4 x\left(8 x^{3}-1\right)}{\left(4 x^{3}+1\right)^{2}} u^{\prime}(x)+u(x)=1
$$

with the unique solution 1 and $\varphi_{2}=1 / x$. The final result is

$$
Y=-4 \sqrt{x} \int \frac{1}{x} \int 0 .
$$

Using Corollary 2 we get the following

Proposition 7 Let (1) have a solution in $\mathcal{A}_{k}$. Let the equation $L y=0$ have linearly independent solutions $\nu_{1}, \ldots, \nu_{l} \in$ $\mathcal{H}_{k}$ and the equation $L y=\psi_{1}$ have a solution $\mu$. Let

$$
\begin{aligned}
& \chi_{1}=\nu_{1}, \chi_{2}=\nabla\left(\nu_{2} / \chi_{1}\right), \ldots, \\
& \chi_{l}=\nabla\left(\ldots \nabla\left(\nu_{l} / \chi_{1}\right) \ldots / \chi_{l-1}\right) \\
& \varphi=\nabla\left(\ldots \nabla\left(\mu / \chi_{1}\right) \ldots / \chi_{l}\right) .
\end{aligned}
$$

Then the equation (6) has a solution in the class of d'Alembertian spaces iff the equation

$$
L^{\left[\chi_{1}\right] . .\left[x_{l}\right][\varphi]} Y=I\left(\psi_{2}, \ldots, \psi_{r}, 0\right)
$$

has a solution in this class. If $I\left(\xi_{1}, \ldots, \xi_{t}, 0\right)$ is a solution of (20) then

$$
I\left(\chi_{1}, \ldots, \chi_{l}, \varphi, \xi_{1}, \ldots, \xi_{t}, 0\right)
$$

is a solution of (6).

Now let the $k$-problem (4) correspond to the equation $L y=$ $\psi$. Let $\hat{u} \in k$ be a particular solution of the $k$-problem and $e_{1}, \ldots, e_{l}$ be a basis of all the solutions belonging to $k$ of the equation

$$
M u=0 .
$$

Then we can set $\nu_{1}=e_{1} \psi, \ldots, \nu_{l}=e_{l} \psi, \mu=\hat{u} \psi$. If $l>0$ then instead of using the formulae (19) it makes sense to compute

$$
v=\frac{\hat{u}}{e_{1}}, w_{1}=\frac{e_{2}}{e_{1}}, \ldots, w_{l-1}=\frac{e_{l}}{e_{1}}
$$

and then compute $\chi_{1}, \ldots, \chi_{l}, \varphi$ as follows:

$$
\begin{aligned}
& \chi_{1}=e_{1} \psi \\
& \chi_{2}=\nabla\left(w_{1}\right), \\
& \chi_{3}=\nabla\left(\nabla\left(w_{2}\right) / \chi_{2}\right), \\
& \ldots \\
& \left.\chi_{l}=\nabla\left(\ldots \nabla\left(\nabla\left(w_{l-1}\right) / \chi_{2}\right) \ldots\right) / \chi_{l-1}\right), \\
& \left.\varphi=\nabla\left(\ldots \nabla\left(\nabla(w) / \chi_{2}\right) \ldots\right) / \chi_{l}\right) .
\end{aligned}
$$

If $l=0$ then $\varphi=\hat{u} \psi$.

The last computational formulae let us formulate the following version of the algorithm:

Algorithm 2 be $\frac{1}{q} B$.

1. If $\operatorname{ord} L=0$, i.e., $L$ is some $q \in k$, then the result will

2. Let $\psi=\operatorname{head}(B)$. Reduce the equation $L y=\psi$ to a $k$-problem. If the $k$-problem has no solution, the algorithm stops with the result "no solution".

3. Let $\hat{u}$ be a solution of the $k$-problem, and $e_{1}, \ldots, e_{l}(l \geq 0)$ a basis of all solutions in $k$ of the equation $M u=0$, which is the homogeneous counterpart of the $k$-problem. Construct $\chi_{1}, \ldots, \chi_{l}$ and $\varphi$ according to (22). If $r=1$ then the result will be $I\left(\chi_{1}, \ldots, \chi_{l}, \varphi, 0\right)$, else apply the algorithm recursively to

$$
L^{\left[\chi_{1}\right] . .\left[\chi_{l}\right][\varphi]}, \operatorname{tail}(B), r-1 .
$$

4. If the result of the recursive call was "no solution" then the final result will be the same. Otherwise, if the result of the recursive call was the d'Alembertian space $I\left(\xi_{1}, \ldots, \xi_{t}, 0\right)$, then the final result will be $I\left(\chi_{1}, \ldots, \chi_{l}, \varphi, \xi_{1}, \ldots, \xi_{t}, 0\right)$.

This version of the algorithm again does not use the search for hyperexponential solutions of equations of the form (9). Consider the differential case. Let

$$
k=\mathbf{C}(x), L=\frac{d^{2}}{d x^{2}}-2 \frac{d}{d x}+1, \mathcal{E}(f)=e^{x} \int 0 .
$$


The equation $L y=e^{x}$ produces the $k$-problem $u^{\prime \prime}=1$. We take $\hat{u}=\frac{1}{2} x^{2}, e_{1}=1, e_{2}=x$. Thus $\chi_{1}=e^{x}, \chi_{2}=1, \varphi=1$ and we get the following solution of equation (6):

$$
e^{x} \int 1 \int 1 \int 0
$$

i.e., the space $e^{x}\left(c_{1} x^{2}+c_{2} x+c_{3}\right)$, where $c_{1}, c_{2}, c_{3}$ are arbitrary constants.

Consider a similar example for the difference case $(k=$ C $(x)$ ). Let $L=\Delta^{2}-2 \Delta+1, \mathcal{E}(f)=\sum 0$ (i.e., $B$ is the field of constants). Then $\psi=1$, the equation $\Delta^{2} y-2 \Delta y+1=1$ produces the $k$-problem $\Delta^{2} u=1$. We take $\hat{u}=x^{2} / 2, e_{1}=$ $1, e_{2}=x$. It gives $\chi_{1}=1, \chi_{2}=1, \varphi=2$ and we get the solution of equation (6)

$$
1 \sum 1 \sum 2 \sum 0
$$

which is equal to $1 \sum 1 \sum 1 \sum 0$, i.e to the space of all polynomials of the degree $\leq 2$.

The following example shows, that in some cases an existing solution of the equation (6) is not found with the help of the considered algorithm. Let in the differential case

$$
k=\mathbf{C}(x), L=\frac{d}{d x}, \mathcal{E}(f)=e^{-x^{2}} \int 0 .
$$

Obviously the equation $y^{\prime}=e^{-x^{2}}$ has no solution in $\mathcal{H}_{k}$ (the corresponding $k$-problem $u^{\prime}-2 x u=1$ has no rational solution). But, if we use the fact that the equation $L y=0$ has the hyperexponential solution $y=1$ then formula (17) gives us the solution

$$
\int e^{-x^{2}} \int 0
$$

of equation (6).

If one uses Algorithm 2 the finding of a solution of (6) is guaranteed in the situation when (9) has no solution in $\mathcal{H}_{k}$. Consider the situation when (1) has a solution $a \in \mathcal{A}_{k}$ such that $h(a)=h(f)$. Here $L \mathcal{E}(a)=\mathcal{E}(f)$ because obviously $\mathcal{E}(f) \subseteq L \mathcal{E}(a)$ and $\operatorname{dim} L \mathcal{E}(a) \leq \operatorname{dim} \mathcal{E}(f)$. Therefore (6) has a solution $A=\mathcal{E}(a)$ such that $\operatorname{dim} A=\operatorname{dim} \mathcal{E}(f)$.

Proposition 8 Let (6) have a solution $A$ in the class of d'Alembertian spaces and $\operatorname{dim} A=\operatorname{dim} \mathcal{E}(f)$. Then the equation $L y=\psi_{1}$ has a solution in $A \cap \mathcal{H}_{k}$.

Proof: Let $L \varphi_{1}=\psi_{1}, \varphi_{1} \in A$. We have $I\left(\psi_{1}, 0\right) \subset L \mathcal{E}\left(\varphi_{1}\right)$ and $L \mathcal{E}\left(\varphi_{1}\right) \subset I\left(\psi_{1}, 0\right)$ (since $\mathcal{E}\left(\varphi_{1}\right) \subset A \cap\left\{d \mid d \in \mathcal{A}_{k}, L d \in\right.$ $\left.\left.I\left(\psi_{1}, 0\right)\right\}\right)$. Therefore $I\left(\psi_{1}, 0\right)=L \mathcal{E}\left(\varphi_{1}\right)$. Now taking into account that $\operatorname{dim} A>\operatorname{dim} \mathcal{E}(f)$, we have $\operatorname{dim} \mathcal{E}\left(\varphi_{1}\right)=1$.

If $\varphi_{1}$ is the unique solution in $\mathcal{H}_{k}$ of the equation $L y=\psi_{1}$ then we can consider the equation $L^{\left[\varphi_{1}\right]} Y=I\left(\psi_{2}, \ldots, \psi_{r}, 0\right)$ and proceed to the search for $\varphi_{2}$ and so on. But if the equation $L y=\psi_{1}$ has other solutions in $\mathcal{H}_{k}$ then we can meet difficulties in the process of constructing $A$ of the same dimension as $\mathcal{E}(f)$. Consider, for example, the equation $y^{\prime}=$ $\ln x$ :

$$
k=\mathbf{C}(x), L=\frac{d}{d x}, \mathcal{E}(f)=1 \int \frac{1}{x} \int 0 .
$$

Then the equation $L y=1$ leads to the $k$-problem $u^{\prime}=1$; if we take $\varphi_{1}=x$ then the second step will give the $k$-problem $x u^{\prime}+u=1$ with the solution $u=1$, and we obtain the solution $x \int \frac{1}{x} \int 0$ of the original equation in the class of d'Alembertian spaces. But if we take $\varphi_{1}=x+1$ then the second step will give the $k$-problem $(x+1) u^{\prime}+\frac{x-1}{x} u=1$ which has no rational solution.

Fortunately Algorithm 2, in the situation where $L y=\varphi_{1}$ has not unique solution in $\mathcal{H}_{k}$, reduces the equation $L Y=$ $\mathcal{E}(f)$ to the equation $L^{\left[\chi_{1}\right] \ldots\left[\chi_{l}\right]} Y=\frac{1}{\chi_{1} \cdot \chi_{l}} \mathcal{E}(f)$ which produces the equation $L^{\left[\chi_{1}\right] \cdots\left[\chi_{l}\right]} y=\frac{\psi_{1}}{\chi_{1} \chi_{l}}$ having the unique hyperexponential solution $\varphi$ (see (22)). Therefore if there exists a d'Alembertian space $A, \operatorname{dim} A=\operatorname{dim} \mathcal{E}(f)$, such that $L A=\mathcal{E}(f)$, then finding a solution $\tilde{A}, \operatorname{dim} \tilde{A} \geq \operatorname{dim} \mathcal{E}(f)$ of (6) is guaranteed.

To construct the complete answer to the question whether (6) has a solution in the class of d'Alembertian spaces, or not, it is sufficient (due to Proposition 2) to use the search for hyperexponential solutions of homogeneous equations only once, when we first meet an equation $N y=\psi$ which produces an unsolvable $k$-problem. This time we have to consider the equation $N y=0$, find the corresponding $\lambda_{1}, \ldots, \lambda_{m} \in \mathcal{H}_{k}$ such that $N^{\left[\lambda_{1}\right] .}{ }^{\left[\lambda_{m}\right]} y=0$ has no solution in $\mathcal{H}_{k}$ and pass from the equation $N y=\psi$ to

$$
N^{\left[\lambda_{1}\right] \ldots\left[\lambda_{m}\right]} y=\frac{\psi}{\lambda_{1} \ldots \lambda_{m}} .
$$

This gives us a new version of the algorithm, which together with $L, B$ and $r$ has the additional argument $b$ : if it is guaranteed that the equation $L y=0$ has no hyperexponential solutions, then $b=1$ else $b=0$.

Algorithm 3 be $\frac{1}{a} B$.

1. If ord $L=0$, i.e., $L$ is some $q \in k$, then the result will 2. Let $\psi=$ head $(B)$. Reduce the equation $L y=\psi$ to a $k$-problem. If the $k$-problem has no solution then go to 5 .

3. Let $\hat{u}$ be a solution of the $k$-problem, and $e_{1}, \ldots, e_{l}(l \geq 0)$ a basis of all solutions in $k$ of the equation $M u=0$, which is the homogeneous counterpart of the $k$-problem. Construct $\chi_{1}, \ldots, \chi_{l}$ and $\varphi$ according to (22). If $r=1$ then the result will be $I\left(\chi_{1}, \ldots, \chi_{l}, \varphi, 0\right)$, else apply the algorithm recursively to

$$
L^{\left[\chi_{1}\right] \cdot\left[\chi_{l}\right][\varphi]}, \operatorname{tail}(B), r-1, b .
$$

4. If the result of the recursive call was "no solution" then the final result will be the same. Otherwise, if the result of the recursive call was the d'Alembertian space $I\left(\xi_{1}, \ldots, \xi_{t}, 0\right)$, then the final result will be $I\left(\chi_{1}, \ldots, \chi_{l}, \varphi, \xi_{1}, \ldots, \xi_{t}, 0\right)$.

5. If $b=1$ the algorithm stops with the result "no solution". If $b=0$ then find $\lambda_{1}, \ldots, \lambda_{m} \in \mathcal{H}_{k}$ such that $L^{\left[\lambda_{1}\right] \ldots\left[\lambda_{m}\right]} y=0$ has no solution in $\mathcal{H}_{k}$. Apply the algorithm recursively to

$$
L^{\left[\lambda_{1}\right] \ldots\left[\lambda_{m}\right]}, \frac{1}{\lambda_{1} \ldots \lambda_{m}} B, r, 1 .
$$

6. If the result of recursive call was "no solution" then the final result will be the same. Otherwise, if the result of the recursive call was the d'Alembertian space $I\left(\xi_{1}, \ldots, \xi_{t}, 0\right)$, then the final result will be $I\left(\lambda_{1}, \ldots, \lambda_{m}, \xi_{1}, \ldots, \xi_{t}, 0\right)$.

Algorithm 3 calls an algorithm for finding hyperexponential solutions only when required. Therefore in the general case this algorithm does not give the space of all d'Alembertian elements which have the $L$-image in $B$. But we can construct this space if we revise the fragment "If $r=1$ then the result will be $I\left(\chi_{1}, \ldots, \chi_{l}, \varphi, 0\right)$ " in step 3 
of the algorithm. If $r=1$ and simultaneously $b=1$ then this fragment gives complete solution of the problem; but if $r=1$ and $b=0$ then it is possible to construct additionally $\lambda_{1}, \ldots, \lambda_{m}$ for the operator which we have at this step (as in step 5) and return the answer $I\left(\chi_{1}, \ldots, \chi_{l}, \varphi, \lambda_{1}, \ldots, \lambda_{m}, 0\right)$. This version of the algorithm (Algorithm 4) gives the maximal solution of $(6)$.

Algorithm 4 to search for the space of all d'Alembertian elements which have the $L$-image in $B$ has the advantage over the approach based on formula (17): the homogeneous equation we deal with has in some cases the order less than $n=\operatorname{ord} L$ (and sometimes this order is equal to 0 ). Observe that if this homogeneous equation has the order 1 then its hyperexponential solution can be written directly without using special complicated algorithms.

When the space $A$ such that $L A=\mathcal{E}(f), \operatorname{dim} A=s$ is constructed we need to choose the elements of this space, which are solutions of (1). It can be done by equating the values of $\nabla^{l} y$ and $\nabla^{l} f$ for $l=0, \ldots, s-1$.

\section{Implementation}

Algorithm 2 was implemented in Maple $\mathbf{5 . 3}$ for an arbitrary Ore polynomial ring and includes several procedures. The main procedure dAsolve 2 takes an operator $L$ and the envelope of the right-hand side $B$ and returns (if found) a solution $A$ of (6) in class of d'Alembertian spaces. Consider again equation (18) and a Maple log of the session which solves this equation in the class of d'Alembertian spaces (here the program is set to the differential case, i.e., $\sigma=1, \delta=\theta=d / d x)$

$>\mathrm{L}:=[-\mathrm{x}, 0,1] ; \mathrm{B}:=\mathrm{dA}\left(\left(4 * \mathrm{x}^{\wedge} 3+1\right) / \mathrm{x}^{\sim}(3 / 2), 1 / \mathrm{x}, 0\right)$;

$$
\begin{aligned}
& L:=[-x, 0,1] \\
& B:=\mathrm{dA}\left(\frac{4 x^{3}+1}{x^{3 / 2}}, \frac{1}{x}, 0\right)
\end{aligned}
$$

$>$ dAsolve2(L, B);

$$
\mathrm{dA}\left(-4 \sqrt{x}, \frac{1}{x}, 0\right)
$$

It takes 7 seconds ${ }^{1}$ to find this solution, of which 5 seconds where spent solving two $k$-problems. As can be seen from this session, an Ore polynomial $L$ is represented as a list of coefficients and the d'Alembertian space of the form (5) is represented as $\mathrm{dA}\left(\psi_{1}, \ldots, \psi_{r}, 0\right)$, where $\mathrm{dA}$ is an unevaluated name.

Several auxiliary procedures are needed in order to use this implementation in practical cases. For example, a procedure to construct $\mathcal{E}(b)$ for a given right-hand side $b$ (we use a partial algorithm that can prompt the user for input), a procedure to choose those solutions from $A$ which satisfy (1) (it is done by equating the values of $\nabla^{s} y$ and $\nabla^{s} f$ for $s=0, \ldots, r-1$ at a point $p$, which is nonsingular for the equations considered, and then by solving a system of linear algebraic equations) and so on.

Before using this program it needs to be set to a concrete $k[\theta]$ by pointing out the independent variable and the concrete $\delta, \sigma$. Additionally it is necessary to provide the program with the full name (including path) of a procedure

${ }^{1}$ All the timings reported in this paper were obtained on $66 \mathrm{Mh}$ IBM PC 486DX.

to solve $k$-problems. In the standard cases, such as differential, difference, $q$-difference and so on, this adjustment is hidden from user and he needs only to select the appropriate standard case. After this is done, the user can call the procedure dAsolver which takes an operator $L$ and a righthand side $b$ and returns a d'Alembertian solution of equation (1) if it was found. This procedure uses the auxiliary procedures mentioned above and the main solver in the class of d'Alembertian spaces dAsolve2

Consider an example. The equation

$$
y^{\prime \prime}-x y=\frac{\left(4 x^{3}+1\right) \ln (x)}{x * \sqrt{x}}
$$

has the elementary particular solution $-4 \sqrt{x} \ln (x)$. The procedure dsolve from Maple $\mathbf{5 . 3}$ gives a particular solution expressed in terms of integrals and Bessel functions and spends 48 seconds searching for that solution:

$$
\begin{aligned}
& >\text { eq: }=\operatorname{diff}(\operatorname{diff}(y(x), x), x)-x * y(x)= \\
& (4 * x-3+1) * \ln (x) /(x * \operatorname{sqrt}(x)) \text { : } \\
& >\mathrm{z} \text { : =dsolve (eq, } \mathrm{y}(\mathrm{x})) \text {; } \\
& \begin{aligned}
z:= & \mathrm{y}(x)=\int \ln (x)\left(4 x^{3}+1\right) \% 1 /\left(x^{5 / 2}(\right. \\
& \% 1 \text { BesselI }\left(\frac{4}{3}, \frac{2}{3} x^{3 / 2}\right) \\
& \left.\left.+ \text { BesselK }\left(\frac{4}{3}, \frac{2}{3} x^{3 / 2}\right) \% 2\right)\right) d x \sqrt{x} \\
& \% 2-\int \ln (x) \% 2\left(4 x^{3}+1\right) /\left(x^{5 / 2}(\right. \\
& \% 1 \text { BesselI }\left(\frac{4}{3}, \frac{2}{3} x^{3 / 2}\right) \\
& \left.\left.+ \text { BesselK }\left(\frac{4}{3}, \frac{2}{3} x^{3 / 2}\right) \% 2\right)\right) d x \sqrt{x} \\
& \% 1+\text { C } 1 \sqrt{x} \% 2+. C 2 \sqrt{x} \% 1 \\
& \% 1:=\text { BesselK }\left(\frac{1}{3}, \frac{2}{3} x^{3 / 2}\right) \\
& \% 2:=\text { BesselI }\left(\frac{1}{3}, \frac{2}{3} x^{3 / 2}\right)
\end{aligned}
\end{aligned}
$$

Below we give the log of a Maple session which uses our program to find a particular solution of this equation:

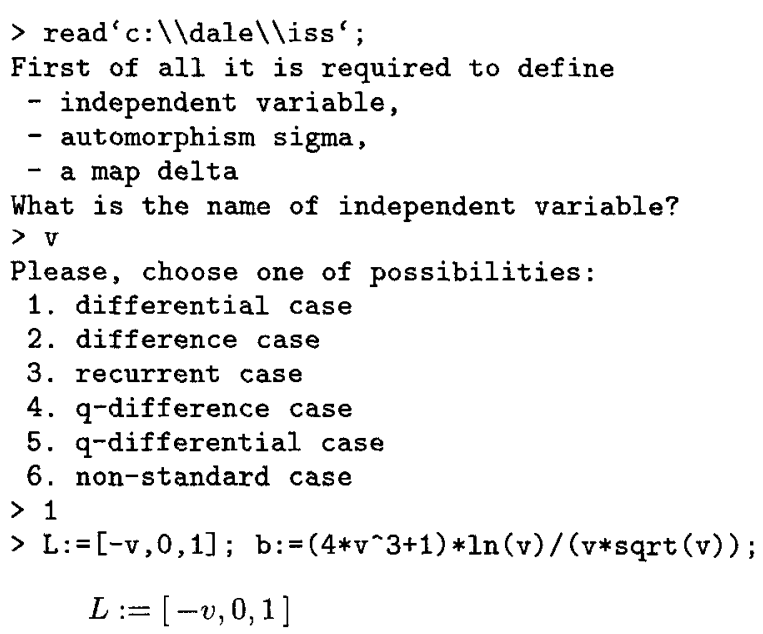


$b:=\frac{\left(4 v^{3}+1\right) \ln (v)}{v^{3 / 2}}$

$>$ dAsolver $(\mathrm{L}, \mathrm{b})$;

$$
-4 \sqrt{v} \ln (v)
$$

Our program spent 12 seconds to produce this answer. In particular, 2 seconds were spent on constructing the envelope of the right-hand side, 7 seconds - on the search for solution in the class of d'Alembertian spaces (of which 5 seconds were spent in order to solve two $k$-problems) and 3 seconds - to choose a particular solution of the equation. To solve $k$-problems in differential case we use M.Bronstein's package ratlode from the Maple share library.

We do not use a general toolkit to handle Ore polynomials such as T.Mulders' package. Algorithms described in this paper do not need operations in $k[\theta]$ more complicated then division by a linear polynomial or multiplication of two polynomials. It allows us to implement our algorithms more efficiently.

We remark that there is an advanced theory concerning inhomogeneous linear ordinary differential equations ([10]). But this theory does not consider, for example, difference and $q$-difference equations, and is not as elementary as the one proposed in this paper.

\section{A remark about the representation of results}

The final result of Algorithms 2-4 could be represented as the pair $(a, H)$ where $a$ is a solution of (1) and $H$ is a d'Alembertian space of solutions of (9). Observe that using, for example, Algorithm 2 we get hyperexponential solutions of homogeneous equations when consider different $k$-problems. We have to transform all these solutions into solutions of (9) and to construct $H$.

Proposition 9 Let $\varphi \in \mathcal{H}_{k}, L \varphi \neq 0$. Let $v \in \mathcal{A}_{k}, L^{[\varphi]} v=$ 0 . Then there exists $w \in \mathcal{A}_{k}$ such that $\nabla(w / \varphi)=v, L w=0$. Proof: We consider the operator $\bar{T}$ defined by (10). Then

$$
\frac{1}{L \varphi} L \circ \varphi=\frac{1}{L \varphi} \bar{T} \nabla+1 \text {. }
$$

Let

$$
z=-\frac{1}{L \varphi} \bar{T} v
$$

Applying $\nabla$ to the both sides of (23) and taking into account that $L^{[\varphi]} v=0$, we obtain $\nabla z=v$. Substituting $\nabla z$ for $v$ into (23) we obtain

$$
\frac{1}{L \varphi} \bar{T} \nabla z+z=0
$$

i.e. $\frac{1}{L \varphi} L \varphi z=0$. Hence we can take $w=\varphi z$, that is

$$
w=-\frac{\varphi}{L \varphi} \bar{T} v
$$

Corollary 4 If we know a space $C$ of solutions of $L^{[\varphi]} y=0$ then we can construct the space $\frac{\varphi}{L \varphi} \vec{T} C$ of solutions of $L y=$ 0 .

The last corollary can be used repeatedly.

\section{Acknowledgements}

We would like to thank Manuel Bronstein, Marko Petkovšek and ISSAC referees who provided us with useful comments on earlier versions of the paper.

\section{References}

[1] Aвramov S.A. (1989): Rational solutions of linear differential and difference equations with polynomial coefficients, USSR, Comput. Math. Math. Phys., 29, 7-12 (translated from $\mathrm{Zh}$. vychisl. mat. mat. fiz. 29, 1611$1620)$.

[2] Abramov S.A., KVaschenko K.YU. (1991): Fast algorithms to search for the rational solutions of linear differential equations with polynomial coefficients, in the Proceedings of ISSAC' 91 , Bonn, Germany, ACM Press, 267-270.

[3] Abramov S.A., Petkovšek M. (1994): D'Alembertian Solutions of Linear Differential and Difference Equations, in the Proceedings of of ISSAC'94, Oxford, UK, ACM Press, 169-174.

[4] ABRAmov S.A. (1995): Rational solutions of linear difference and $q$-difference equations with polynomial coefficients, in the Proceedings of ISSAC'95, Montreal, Canada, ACM Press, 285-289.

[5] Abramov S., Bronstein M. \& Petkovšek M. (1995): On polynomial solutions of linear operator equations, in the Proceedings of ISSAC'95, Montreal, Canada, ACM Press, 290-296.

6] Abramov S.A., Petkovšek M. (1995): Finding all $q$-hypergeometric solutions of $q$-difference equations, $\mathrm{B}$. Leclerc and J.-Y. Thibon, eds., Proc. FPSAC '95 (Univ. de Marne-la-Vallée, Noisy-le-Grand) 1-10.

[7] Abramov S.A. (1996): Symbolic algorithms to find particular d'Alembertian solutions of linear equations, Programming and Computer Software (translated from Programmirovanie, N 1, 39-50).

[8] BRONSTEIN M. (1992): On solutions of linear ordinary differential equations in their coefficient field, Journal of Symbolic Computation, 13, 413-439.

[9] Bronstein M., Petkovšek M. (1996): An Introduction to Pseudo-Linear Algebra, Theoretical Computer Science, 157 (in press).

[10] Davenport J.H., Singer M.F. (1986): Elementary and Liouvillian solutions of linear differential equations, Journal of Symbolic Computation, 1986, 2, 237260.

[11] ORE O. (1933): Theory of non-commutative polynomials, Annals of Mathematics 34, 480-508.

[12] PETKovšEK M. (1992): Hypergeometrıc solutions of linear recurrences with polynomial coefficients, J. Symb. Comput. 14, 243-264.

Sergei A. Abramov is a Leading Investigator in the Computer Center of the Russian Academy of Sciences and a Professor in the Moscow State University. Prof. Abramov earned a Ph.D. in Computer Science (1972) and a Doctor of 
Mathematics (1983) from the Computer Center of the Russian Academy of Sciences and the Moscow State University respectively. His research interests are in computer algebraic aspects of the theory of differential and difference equations.

Eugene V. Zima is an Associate Professor in the Moscow State University, and a Senior Researcher in the Computer Center of the Russian Academy of Sciences. Prof. Zima earned a Ph.D. in Computer Science (1985) from the Moscow State University. His research interests include Computer Algebra, Symbolic-Numeric Interface, Program Optimization, Parallel Computations. 